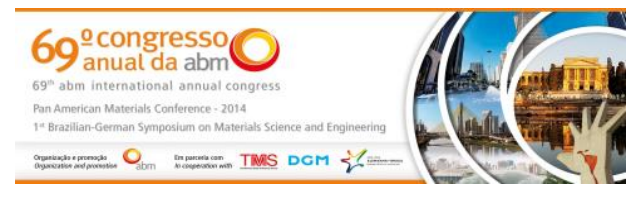

Tema: Produtos Metálicos não-ferrosos

\title{
ANÁLISE DA INFLUÊNCIA DA INSERÇÃO DE TEORES DE Ni SOBRE AS CARACTERÍSTICAS ESTRUTURAIS E AS PROPRIEDADES MECÂNICAS E ELÉTRICAS DE UMA LIGA Al-0,05\%Cu-[0,24-0,28]\%Fe-0,7\%Si*
}

\author{
Amanda Thicyane de Miranda Duarte de Souza ${ }^{1}$ \\ Isabella Cristina da Silva Jorge ${ }^{2}$ \\ Jessiana Avelar de Lima $^{3}$ \\ Clóvis larlande Oliveira Santana ${ }^{4}$ \\ Patrick dos Santos Nogueira ${ }^{5}$ \\ José Maria do Vale Quaresma ${ }^{6}$
}

\section{Resumo}

A crescente demanda de energia elétrica em nosso país sobrecarrega o Sistema de Transmissão [Tx] e Distribuição [Dx], e gera a necessidade de constantes ampliações para não prejudicar o desenvolvimento do Brasil. Neste contexto foram realizados estudos visando avaliar o efeito da adição de três teores de $\mathrm{Ni}$ em uma liga $\mathrm{Al}-0,05 \% \mathrm{Cu}-[0,24-0,28] \% \mathrm{Fe}-0,7 \% \mathrm{Si}$ destinada à produção de cabos elétricos. $\mathrm{O}$ estudo parte da verificação e posterior comparação das características estruturais, mecânicas e elétricas da liga com a adição do elemento citado. Para a realização do mesmo, os perfis estudados foram obtidos por solidificação estática direta em lingoteira metálica e as amostras foram obtidas a partir de perfis solidificados, usinados e deformados a frio para diferentes diâmetros. Nestas circunstâncias, o diâmetro de $3,0 \mathrm{~mm}$ foi aquele que apresentou as melhores respostas elétricas, mecânicas e estruturais, sendo, portanto, adotado como fonte de estudo.

Palavras-chave: Níquel; Propriedades elétricas; Propriedades Estruturais; Propriedades mecânicas.

\section{ANALYSIS OF INFLUENCE OF ADDITION OF Ni CONTENTS ON STRUCTURAL CHARACTERISTICS AND ELECTRICAL AND MECHANICAL PROPERTIES FOR Al- $0.05 \% \mathrm{Cu}-[0.24$ to 0.28$] \% \mathrm{Fe}-0.7 \% \mathrm{Si}$ ALLOY}

\section{Abstract}

The growing demand for electricity in our country overloads the Transmission [Tx] and Distribution [Dx] System, and generates the need for constant expansions in order not to prejudice the Brazil's development. In this context, studies were conducted to evaluate the effect of the addition of three $\mathrm{Ni}$ contents in an $\mathrm{Al}-0.05 \% \mathrm{Cu}-[0.24$ to 0.28$] \% \mathrm{Fe}-0.7 \% \mathrm{Si}$ alloy aimed at producing electrical cables. The study begins with verification and subsequent comparison of the structural, mechanical and electrical characteristics of the alloy with the addition of the element mentioned. For its realization, the studied profiles were obtained by direct casting in a metallic mold and samples were obtained from solidified, machined and cold deformed profiles for different diameters. In these circumstances, the diameter of 3.0 $\mathrm{mm}$ was one that showed the best electrical, mechanical and structural responses, and therefore, adopted as a source of study.

Keywords: Nickel; Electrical properties; Structural properties; Mechanical properties.

1 Engenheira Mecânica, Mestranda em Engenharia Mecânica, Instituto de Tecnologia, Universidade Federal do Pará, Belém (UFPA), PA, Brasil.

2 Mestre em Engenharia Mecânica, Doutoranda em Eng. de Materiais, Depto. Eng. de Materiais e Processos Químicos e Metalúrgicos, Instituto de Tecnologia, Pontifícia Universidade Católica do Rio de Janeiro (PUCRio), Rio de Janeiro, RJ, Brasil.

3 Engenheira de Produção, Mestranda em Eng. Mecânica, Instituto de Tecnologia, UFPA, Belém, PA, Brasil.

4 Graduando em Engenharia Mecânica, Instituto de Tecnologia, UFPA, Belém, PA, Brasil.

5 Engenheiro Mecânico, Instituto de Tecnologia, Universidade Federal do Pará, Belém, PA, Brasil.

6 Engenheiro Mecânico, Doutor em Eng. Mecânica, Instituto de Tecnologia, UFPA, Belém, PA, Brasil.

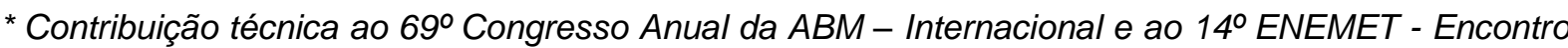
Nacional de Estudantes de Engenharia Metalúrgica, de Materiais e de Minas, 21 a 25 de julho de 2014, São Paulo, SP, Brasil.
} 


\section{INTRODUÇÃO}

Em 2010, e de acordo com o IBGE, havia 1,3\% de domicílios sem energia elétrica no Brasil. Nas áreas rurais, esta porcentagem chegava a 7,4\%. O programa de eletrificação rural do Governo Federal "Luz para Todos", que já beneficiou cerca de 14 milhões de brasileiros, foi um propulsor do aumento do consumo de cabos de alumínio [1].

Embora a eletricidade seja responsável por uma revolução no cotidiano da humanidade, atribui-se à concepção dos fios e cabos os avanços relacionados à transmissão e distribuição de energia elétrica. E quanto à produção destes, os metais mais utilizados são - desde o início - o cobre e o alumínio. E embora o cobre possua resistência elétrica menor e maior maleabilidade em relação ao alumínio, este último ainda tem a vantagem de ser mais leve e comumente empregado em linhas aéreas de distribuição e transmissão, porém com uma dimensão maior, permitindo que os vãos entre as torres sejam maiores e necessitando - portanto de um menor número destes elementos na linha, o que, por sua vez, promove redução de custos de instalação [2].

Portanto, é considerando o cenário acima descrito que o Grupo de Pesquisa em Engenharia de Materiais - GPEMAT da Universidade Federal do Pará (UFPA) desenvolve desde 2007 trabalhos ao combinar e aperfeiçoar as características dos materiais à base de alumínio, pois os investimentos realizados para modificar as linhas de transmissão já existentes com os cabos produzidos a partir destes novos materiais seriam bem menores se comparados aos atuais custos de manutenção das linhas já existentes. Existindo ainda, para estes novos materiais, os incrementos de temperatura operacional, sem aumentar as perdas de Efeito-Joule, enquanto limita a flecha entre as torres dentro de valores aceitáveis nas instalações existentes.

Assim sendo, o presente trabalho visa à caracterização mecânica, elétrica e estrutural da liga Al-0,05\%Cu-[0,24-0,28]\%Fe-0,7\%Si modificada com os diferentes teores de $\mathrm{Ni}(0,010 ; 0,025$ e 0,040$) \%$ a fim de avaliar os efeitos dos mesmos sobre as propriedades dos materiais passíveis de serem empregados na fabricação de cabos destinados à Transmissão [Tx] e Distribuição [Dx] de energia elétrica. E para a realização deste trabalho, os perfis estudados foram obtidos por solidificação estática direta em lingoteira metálica e as amostras foram obtidas a partir de perfis solidificados, usinados e deformados a frio para diferentes diâmetros. $E$ sob tais circunstâncias, o diâmetro de $3,0 \mathrm{~mm}$ destaca-se com as melhores respostas elétricas, mecânicas e estruturais, motivo pelo qual foi adotado como fonte de estudo.

E para posterior análise dos resultados abordados neste, faz-se necessário estabelecer alguns breves conceitos sobre fratura e metalografia quantitativa.

Segundo Bresciane et al. [3], a fratura de um corpo sólido - que está ligada às tensões aplicadas sobre um material quando as mesmas excedem a capacidade de carga que o elemento suporta - consiste na separação desse corpo em duas ou mais partes sob a ação de esforços mecânicos. Ainda segundo o referido autor, os materiais de engenharias podem ser divididos em duas modalidades, frágil e dúctil.

E de acordo com Reed-Hill [4], fratura frágil é aquela na qual o movimento da trinca envolve uma deformação plástica muito pequena do metal e a ela adjacente. Em sua topografia não há qualquer sinal de deformação plástica generalizada, e em alguns casos, somente linhas ou nervuras que irradiam a partir do ponto de origem da trinca e se propagam em forma de leque (Figura 1(b)).

\footnotetext{
* Contribuição técnica ao $69^{\circ}$ Congresso Anual da ABM - Internacional e ao 14ํㅡㄹ ENEMET - Encontro Nacional de Estudantes de Engenharia Metalúrgica, de Materiais e de Minas, 21 a 25 de julho de 2014, São Paulo, SP, Brasil.
} 

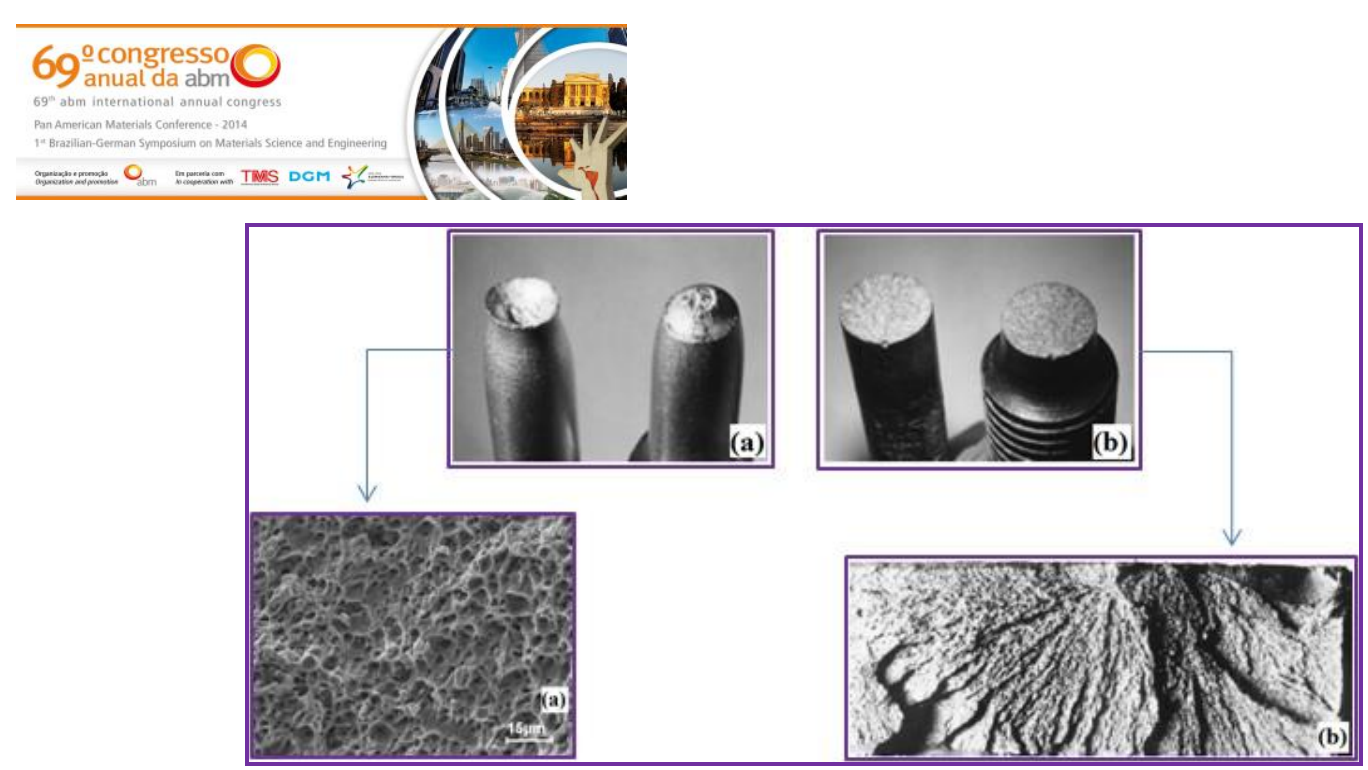

Figura 1. Tipos de fratura: (a) fratura dúctil; (b) fratura frágil.

Já os materiais dúcteis, objeto de estudo deste trabalho, apresentam, tipicamente, antes da fratura uma deformação plástica substancial com uma grande absorção de energia, já que é necessária uma maior energia de deformação para induzir a fratura dada a boa tenacidade destes materiais. E o tipo de fratura dúctil mais comum em ensaios de tração é aquele em que a fratura é precedida por uma quantidade moderada de empescoçamento do material, seguida de nucleação, crescimento e coalescência de microcavidades (ou dimples em inglês) dentro da amostra (Figura 1 (a)). Tais estruturas se formam quando uma tensão elevada causa uma fratura localizada nos contornos de grão ou nas interfaces entre o metal e pequenas inclusões. Quanto mais a tensão local aumenta, mais as microcavidades crescem e coalescem formando cavidades maiores. Então, quando a seção resistente é reduzida demais para suportar a carga presente, a fratura ocorre [5].

Quanto à metalografia quantitativa, esta - aplicada às Ciências dos Materiais - é utilizada para encontrar uma relação simples entre as propriedades de um metal e a sua microestrutura que procure exprimir, em termos precisos, determinados fatores ou parâmetros estruturais. Sem tal possibilidade, a apreciação desses parâmetros não pode ser senão qualitativa e, consequentemente, de interesse limitado. Neste contexto, a metalografia quantitativa fornece meios de quantificar a microestrutura, cujo objetivo é determinar a quantidade, a forma, o tamanho e a distribuição dos constituintes, fases e defeitos [6].

E neste contexto, o trabalho ora proposto objetiva avaliar mecânica, estrutural e eletricamente a liga modificada com os teores de Ni propostos.

\section{MATERIAIS E MÉTODOS}

A Figura 2 contém o fluxograma esquemático de obtenção das ligas desenvolvidas neste trabalho. E para tal, este foi dividido em etapas, as quais serão descritas posteriormente.

As ligas desenvolvidas neste trabalho são visadas para a fabricação de linhas aéreas para transmissão e distribuição de energia elétrica ao passo em que se sabe que as ligas de alumínio para tais fins são desenvolvidas com baixos teores de elementos solutos.

O processo de fundição e solidificação de metais transcorreu por várias etapas, e a confirmação da composição química é uma das principais etapas. O processo de fundição foi iniciado com o corte dos lingotes de alumínio, cedidos pela empresa ALUBAR METAIS S/A e outros elementos para compor da liga. Após a pesagem, as massas dos materiais foram introduzidas em um cadinho de carbeto de silício de $3,5 \mathrm{l}$

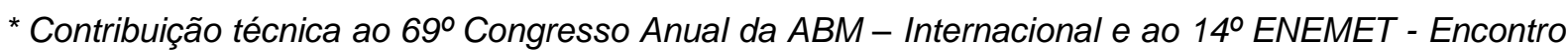
Nacional de Estudantes de Engenharia Metalúrgica, de Materiais e de Minas, 21 a 25 de julho de 2014, São Paulo, SP, Brasil.
} 


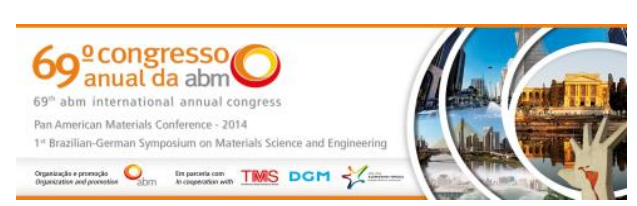

de volume, o qual foi pintado internamente com solução de caulim - para evitar a aderência de uma quantidade considerável de metal - e submetido a um préaquecimento de vinte minutos a $150^{\circ} \mathrm{C}$ para eliminar a umidade.

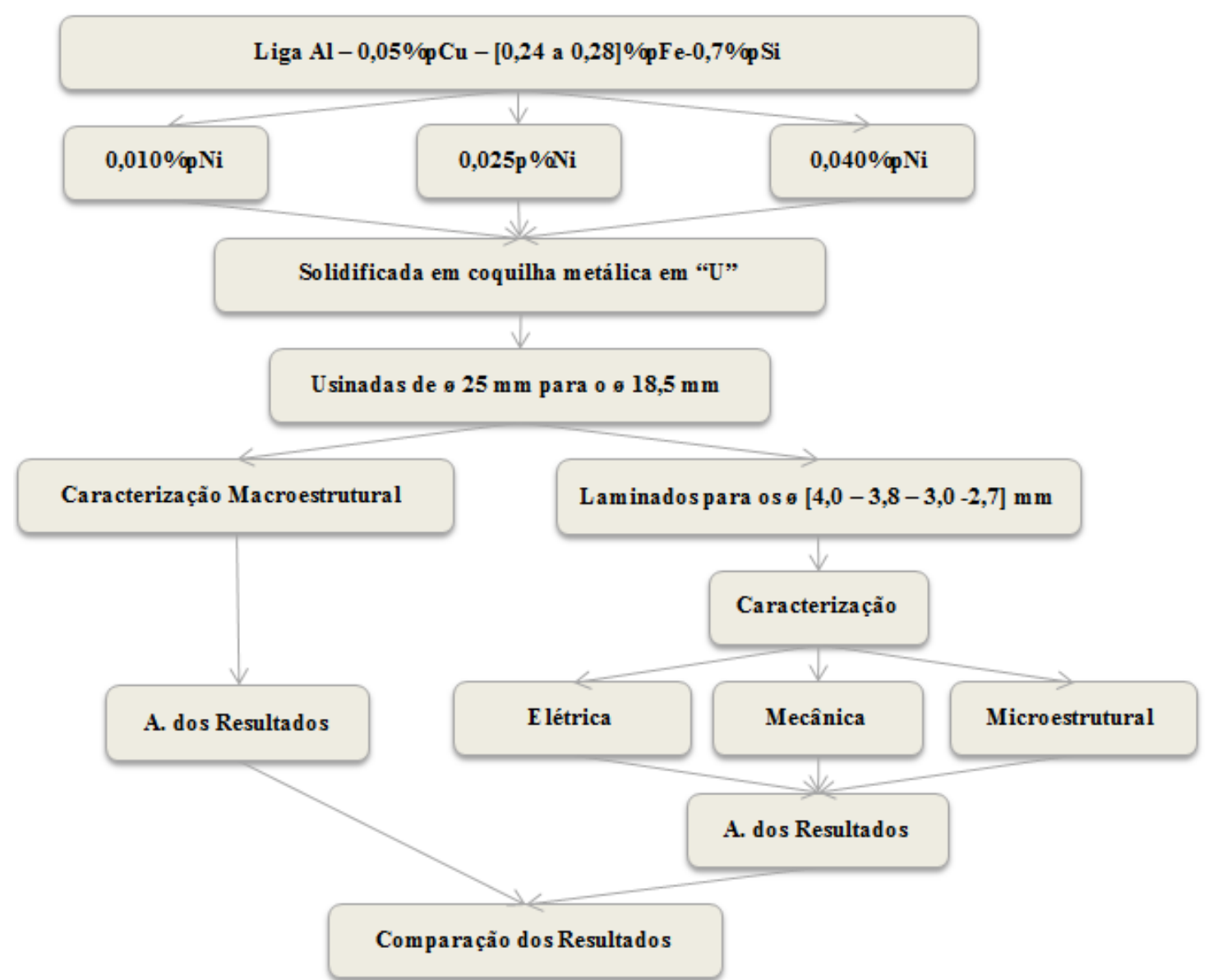

Figura 2. Fluxograma das operações metalúrgicas e de caracterização para a liga base com adição de teores de $\mathrm{Ni}$.

O processo de fundição e solidificação de metais transcorreu por várias etapas, e a confirmação da composição química é uma das principais etapas. O processo de fundição foi iniciado com o corte dos lingotes de alumínio, cedidos pela empresa ALUBAR METAIS S/A e outros elementos para compor da liga. Após a pesagem, as massas dos materiais foram introduzidas em um cadinho de carbeto de silício de 3,5 I de volume, o qual foi pintado internamente com solução de caulim - para evitar a aderência de uma quantidade considerável de metal - e submetido a um préaquecimento de vinte minutos a $150^{\circ} \mathrm{C}$ para eliminar a umidade.

Após a obtenção da liga-base [Al-0,05\%Cu-[0,24 - 0,28]\%Fe-0,7\%Si], a ela foram adicionados os teores de $(0,010 ; 0,025$ e 0,040$) \% \mathrm{Ni}$ (em cadinhos diferenciados). Adotou-se a coquilha de formato em "U" como sistema operacional de solidificação. Logo após o desmolde, os perfis obtidos foram usinados para o diâmetro 18,5 mm, sendo posteriormente submetidos ao processo de deformação por laminação a frio, gerando os corpos de provas em forma de fios, nas dimensões de 4,0;3,8; 3,0 e 2,7 $\mathrm{mm}$, obtendo-se os corpos de prova finais para realizar os ensaios de resistência elétrica e tração.

A microestrutura das ligas foi obtida em corpos de prova resultantes do ensaio de tração que foram preparados para caracterização estrutural realizada via Microscópio Eletrônico de Varredura - MEV, que envolve corte da região da fratura.

* Contribuição técnica ao 69 Congresso Anual da ABM - Internacional e ao 14ํㅡㄹ ENEMET - Encontro Nacional de Estudantes de Engenharia Metalúrgica, de Materiais e de Minas, 21 a 25 de julho de 2014, São Paulo, SP, Brasil. 


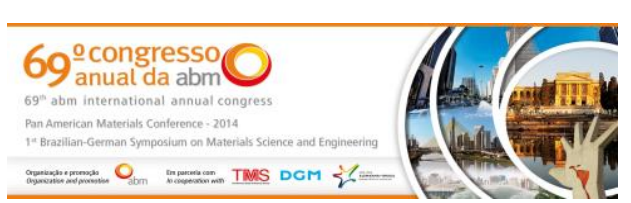

A medição das microcavidades foi realizada de acordo os critérios utilizados por Narayanasamy et al. [7,8], Ravindran et al. [9], Naga Krishna et al. [10]: traça-se duas linhas ( $\mathrm{L}=$ comprimento e $\mathrm{W}=$ largura) perpendiculares entre si que tocam as bordas das microcavidades com o auxílio do software Motic Plus 7.0. Este método possibilita a determinação da razão [L/W] das dimensões das microcavidades, através da Equação 1, com a qual se avalia a forma das microcavidades (esféricas, alongadas ou achatadas).

$$
\vartheta=\frac{L}{W}
$$

Para cada imagem adquirida foram realizadas vinte (20) medições e a partir destas é realizado o cálculo da média e desvio padrão correspondente a cada diâmetro.

Deve-se considerar também o trabalho de conformação mecânica, ao qual todas as ligas desenvolvidas neste trabalho foram submetidas, considerando que as amostras foram usinadas do diâmetro $22,5 \mathrm{~mm}$ para o de $18,5 \mathrm{~mm}$ e, então laminadas em uma sequência de diâmetros de interesse comercial, tais como $\varnothing=[4,0 ; 3,8 ; 3,0$ e $2,7] \mathrm{mm}$. Assim, foi possível construir os gráficos da Figura 3, nos quais se observa, passe a passe, como o material foi deformado na matriz de conformação a frio.

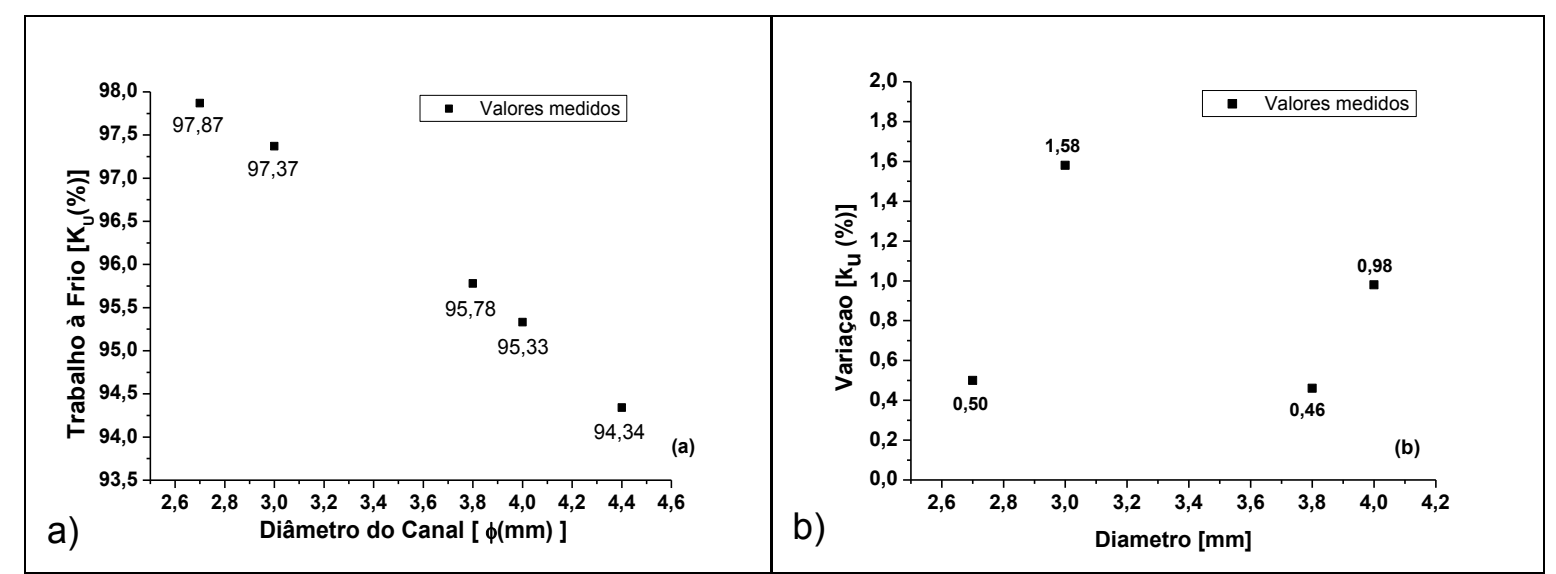

Figura 3. (a) Trabalho a frio previsto; (b) Variação do trabalho a frio previsto, em função dos diâmetros dos canais de laminação, diâmetro inicial igual a $18,5 \mathrm{~mm}$.

\section{RESULTADOS E DISCUSSÃO}

\subsection{Resultados}

\subsubsection{Caracterização microestrutural, mecânica e elétrica}

Os dados abaixo se referem às caracterizações mecânica, microestrutural e elétrica realizadas nas ligas modificadas com Ni. É possível observar nas Figuras (4a, 6a e

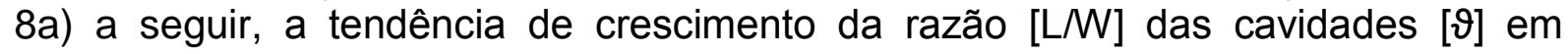
relação ao aumento do diâmetro dos fios $\left[\varnothing_{F}(\mathrm{~mm})\right]$ em estudo. Assim, para as Figuras 4a, 6a e 8a, consideram-se dois aspectos: 1) Independente da composição, o fio com $\varnothing_{\mathrm{F}}=3,0 \mathrm{~mm}$ apresenta sempre a menor razão [L/W] das microcavidades [Э]; 2) Conjunturalmente, os valores para a razão [L/W] das microcavidades [७] apresentam forte tendência de crescimento em relação ao diâmetro dos fios em estudos.

\footnotetext{
* Contribuição técnica ao $69^{\circ}$ Congresso Anual da ABM - Internacional e ao 14ํㅡㄹ ENEMET - Encontro Nacional de Estudantes de Engenharia Metalúrgica, de Materiais e de Minas, 21 a 25 de julho de 2014, São Paulo, SP, Brasil.
} 

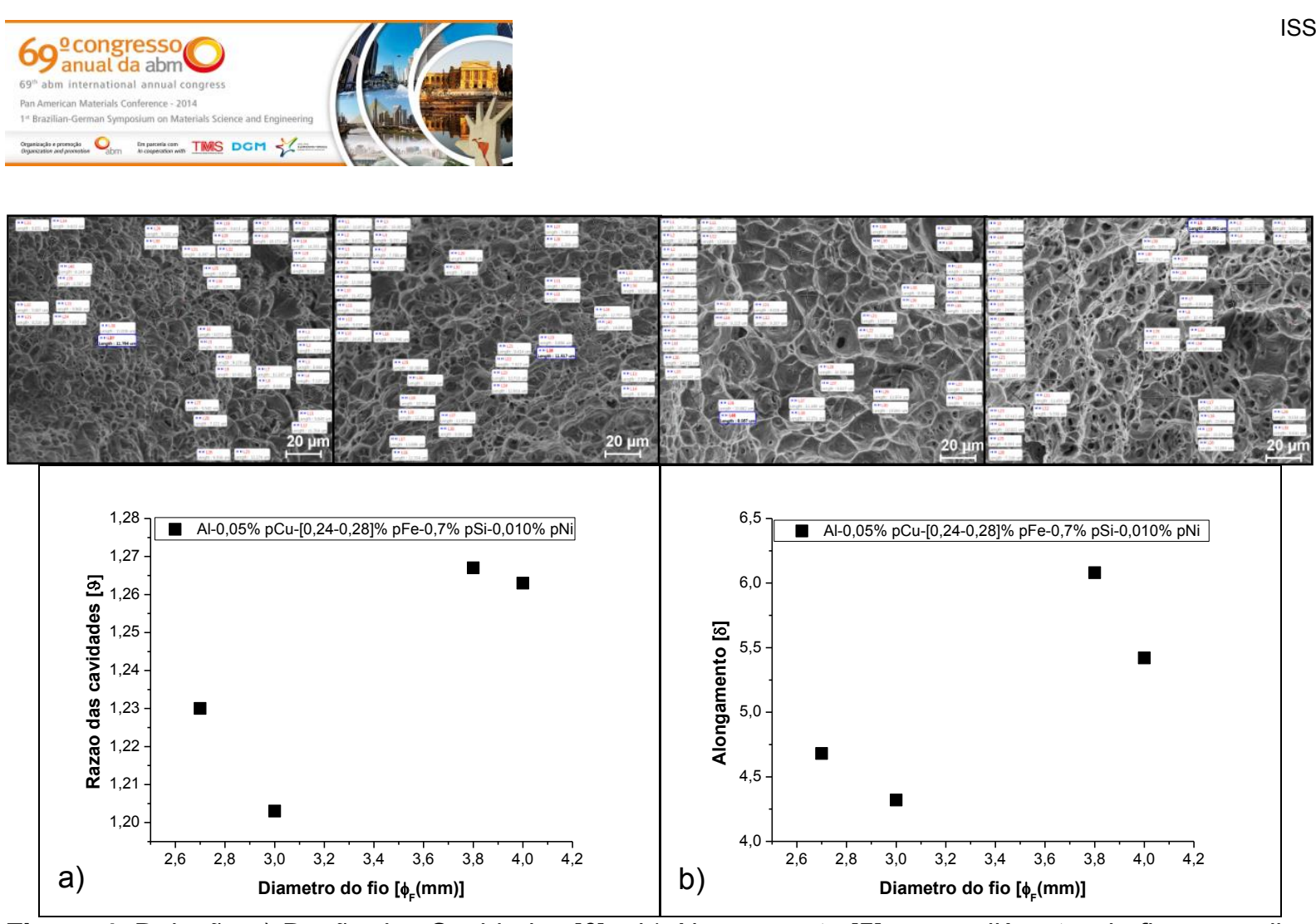

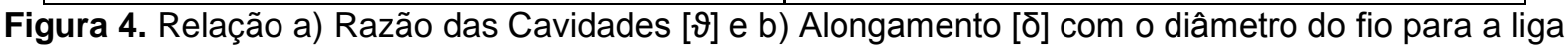
Al-0,05\%Cu-[0,24-0,28]\%Fe-0,7\%Si-0,010\%Ni.

No que diz respeito à ductilidade do material, e para este trabalho, relacionam-se os valores para razão de cavidades e alongamento. Logo, através das Figuras $4 \mathrm{~b}, 6 \mathrm{~b}$ e $8 \mathrm{~b}$, nota-se que o comportamento da evolução para o alongamento apresenta tendência crescente e coerente com o também comportamento de evolução para a razão das cavidades, com destaque para o diâmetro de $3,0 \mathrm{~mm}$, indicando que 0 referido possui maior rigidez, e consequentemente, é mais resistente às deformações, pois a forma e tamanho das microcavidades está relacionada ao alongamento sofrido pelo material [7-10].

Em relação às Figuras 5, 7 e 9, observa-se o mesmo comportamento gráfico para as propriedades mecânicas e elétricas. $E$, neste caso, os aspectos a serem observados são: 1) Independente das composições, os fios com o $\varnothing_{\mathrm{F}}=3,0 \mathrm{~mm}$ sempre apresentam os maiores valores para estas duas propriedades; 2) Conjunturalmente, os valores obtidos para estas duas propriedades apresentam nítida tendência de queda com o aumento do diâmetro do fio.
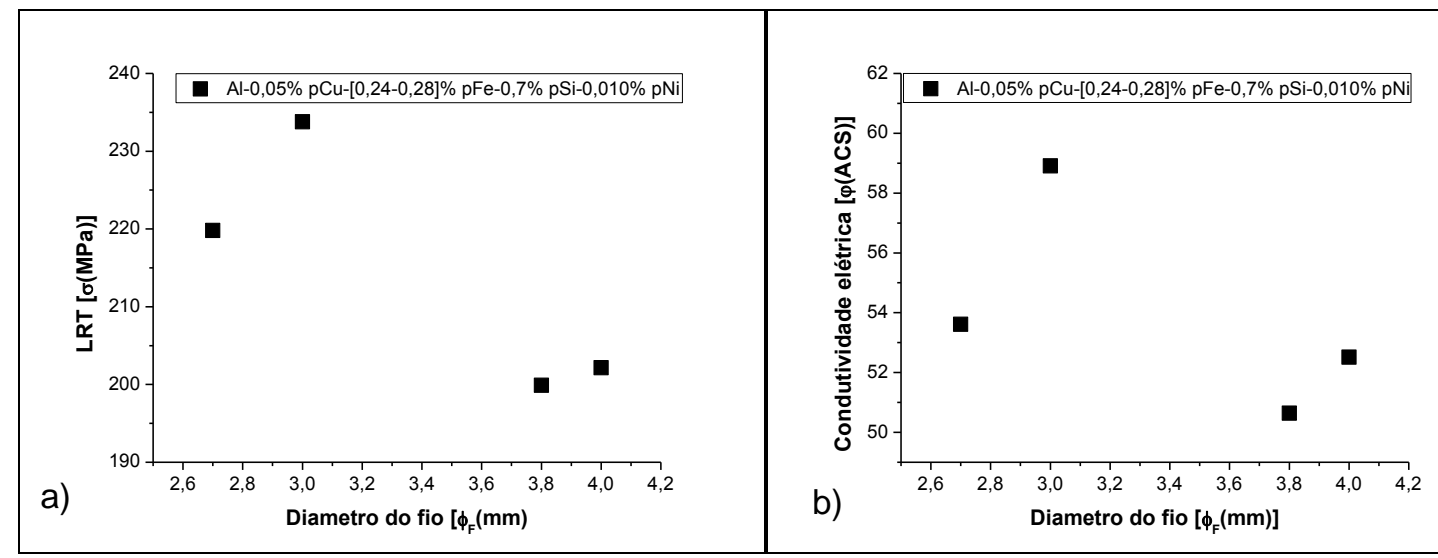

Figura 5. a) Relação de LRT [ $\sigma(\mathrm{MPa})]$ e diâmetro do fio e b) Condutividade Elétrica $[\varphi(\% \mid A C S)]$ e diâmetro do fio para a liga $\mathrm{Al}-0,05 \% \mathrm{Cu}-[0,24-0,28] \% \mathrm{Fe}-0,7 \% \mathrm{Si}-0,010 \% \mathrm{Ni}$ associadas às topografias das fraturas dos diâmetros estudados $(2,7 \mathrm{~mm} ; 3,0 \mathrm{~mm} ; 3,8 \mathrm{~mm}$ e $4,0 \mathrm{~mm}$ respectivamente).

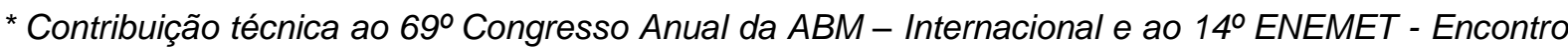
Nacional de Estudantes de Engenharia Metalúrgica, de Materiais e de Minas, 21 a 25 de julho de 2014, São Paulo, SP, Brasil.
} 

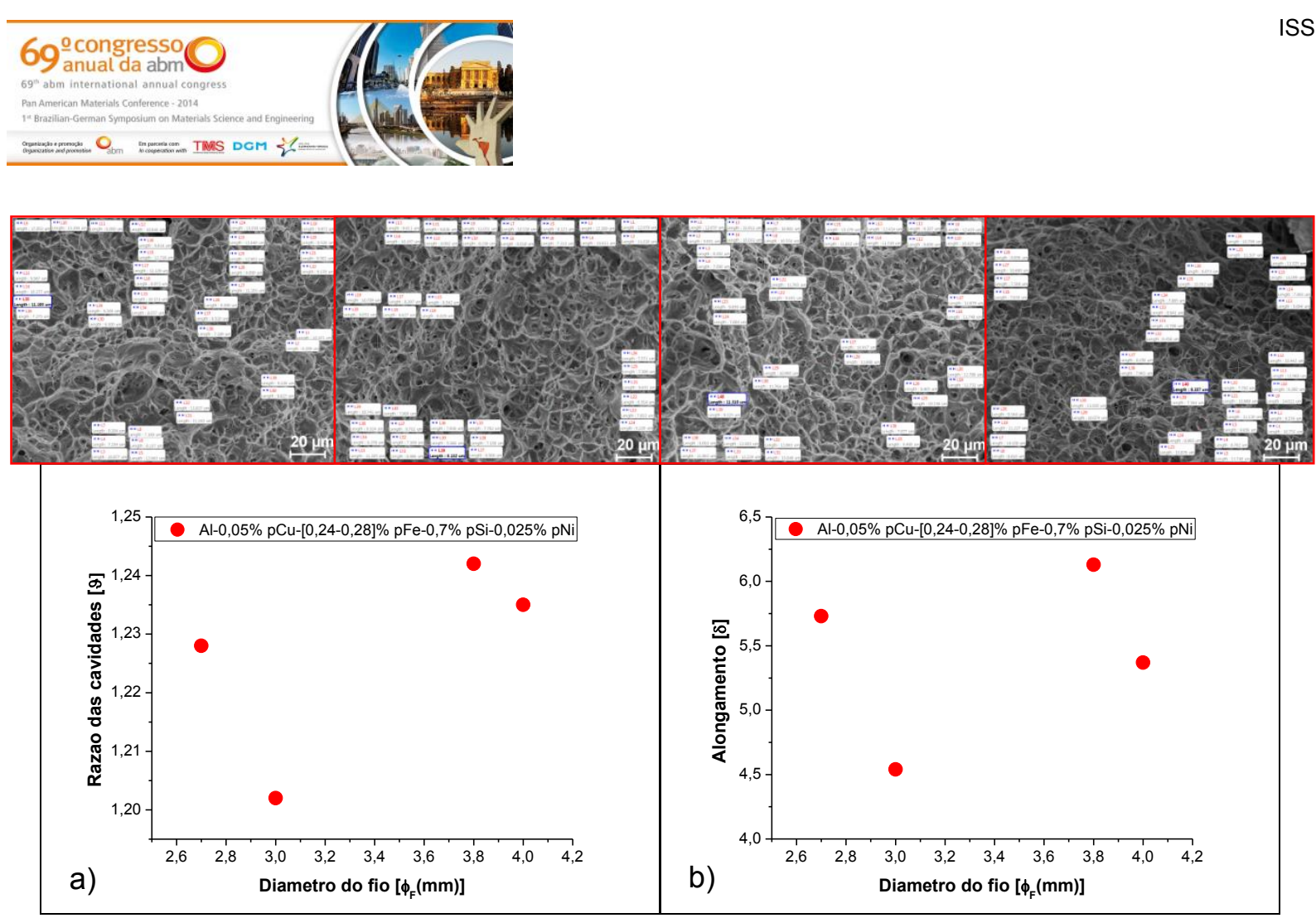

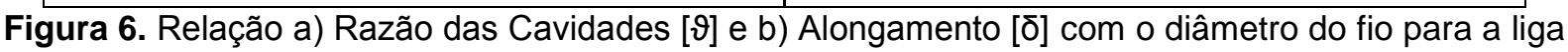
Al-0,05\%Cu-[0,24-0,28]\%Fe-0,7\%Si-0,025\%Ni.

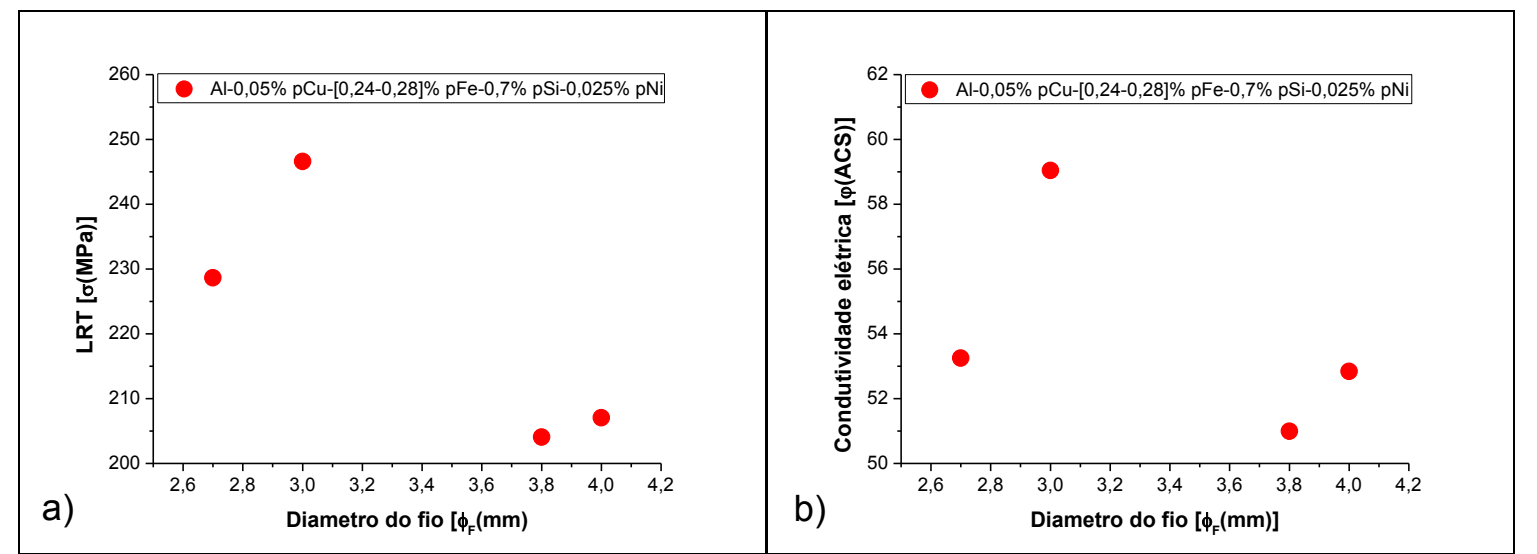

Figura 7. a) Relação de LRT [ $\sigma(\mathrm{MPa})]$ e diâmetro do fio e b) Condutividade Elétrica $[\varphi(\% \mid A C S)]$ e diâmetro do fio para a liga Al-0,05\%Cu-[0,24-0,28]\%Fe-0,7\%Si-0,025\% Ni associadas às topografias das fraturas dos diâmetros estudados $(2,7 \mathrm{~mm} ; 3,0 \mathrm{~mm} ; 3,8 \mathrm{~mm}$ e $4,0 \mathrm{~mm}$ respectivamente).

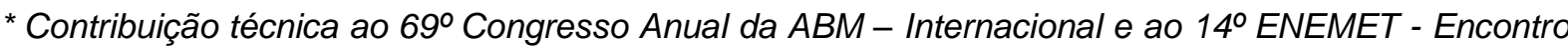
Nacional de Estudantes de Engenharia Metalúrgica, de Materiais e de Minas, 21 a 25 de julho de 2014, São Paulo, SP, Brasil.
} 


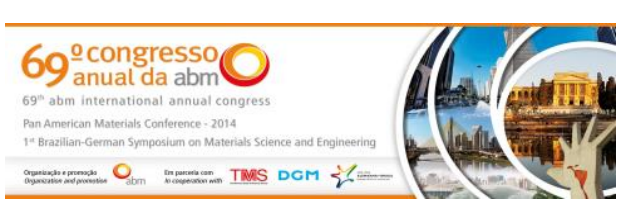

devido ao alto grau de deformação sofrido pelo referido diâmetro, como observado na Figura 3. O comportamento das microcavidades para os fios com os outros diâmetros tendem a coalescência, possível causa dos menores valores para seus LRT.

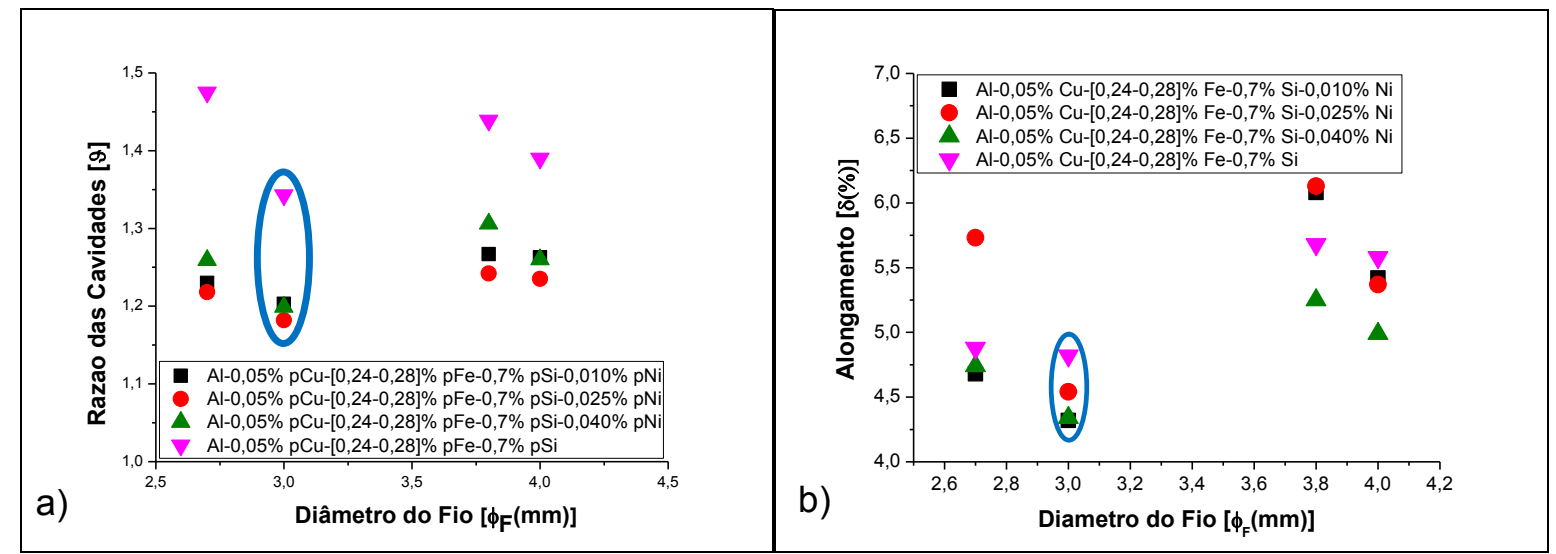

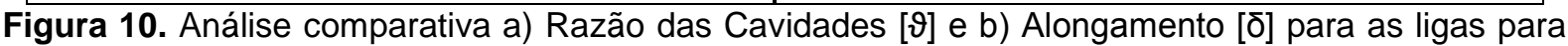
as ligas: Al-0,05\%Cu-[0,24-0,28]\%Fe-0,7\%Si; Al-0,05\%Cu-[0,24-0,28]\%Fe-0,7\%Si-0,010\%Ni; Al0,05\%Cu-[0,24-0,28]\%Fe-0,7\%Si-0,025\%Ni e Al-0,05\%Cu-[0,24-0,28]\%Fe-0,7\%Si-0,040\%Ni.

Os valores dos LRT plotados nas Figuras 11 e 12 permitem a seguinte análise:

1) O comportamento mecânico das ligas, via análise do LRT, pode ser associado tanto ao crescente teor de $\mathrm{Ni}$, quanto ao crescente grau de trabalho a frio submetido, vide Figura 3. Por outro lado, existe a possibilidade do $\mathrm{Ni}$, mesmo nos níveis estudados, contribuir para a formação de partículas de segunda fase capazes de dificultar o caminhar das discordâncias com maior ênfase para os teores [0,025/0,04]\%Ni;

2) O comportamento elétrico das ligas, via análise da condutividade elétrica, permite que se associe a presença destas partículas de segunda fase ao emaranhado das discordâncias e que, em consequência, apresentam resistência ao fluxo de elétrons, com menor ênfase para os teores $[0,01 / 0,025] \% \mathrm{Ni}$;

3) O teor de $0,025 \% \mathrm{Ni}$ apresenta melhor performance, pois ora está associado com o melhor comportamento mecânico, ora está associado ao melhor comportamento elétrico;

4) $O$ fio com $\varnothing_{\mathrm{F}}=3,0 \mathrm{~mm}$ associa elevado LRT com elevado índice de Condutividade Elétrica. O esperado nestas circunstâncias é que os índices de Condutividade Elétrica fossem baixos. É possível que os baixos índices de coalescência das microcavidades [menores razões (L/W)] apresentados por este perfil sejam consequência do fenômeno da Recuperação Dinâmica ajustada à matriz de deformação utilizada na obtenção do mesmo.

\footnotetext{
* Contribuição técnica ao $69^{\circ}$ Congresso Anual da ABM - Internacional e ao 14ํㅡㄹ ENEMET - Encontro Nacional de Estudantes de Engenharia Metalúrgica, de Materiais e de Minas, 21 a 25 de julho de 2014, São Paulo, SP, Brasil.
} 

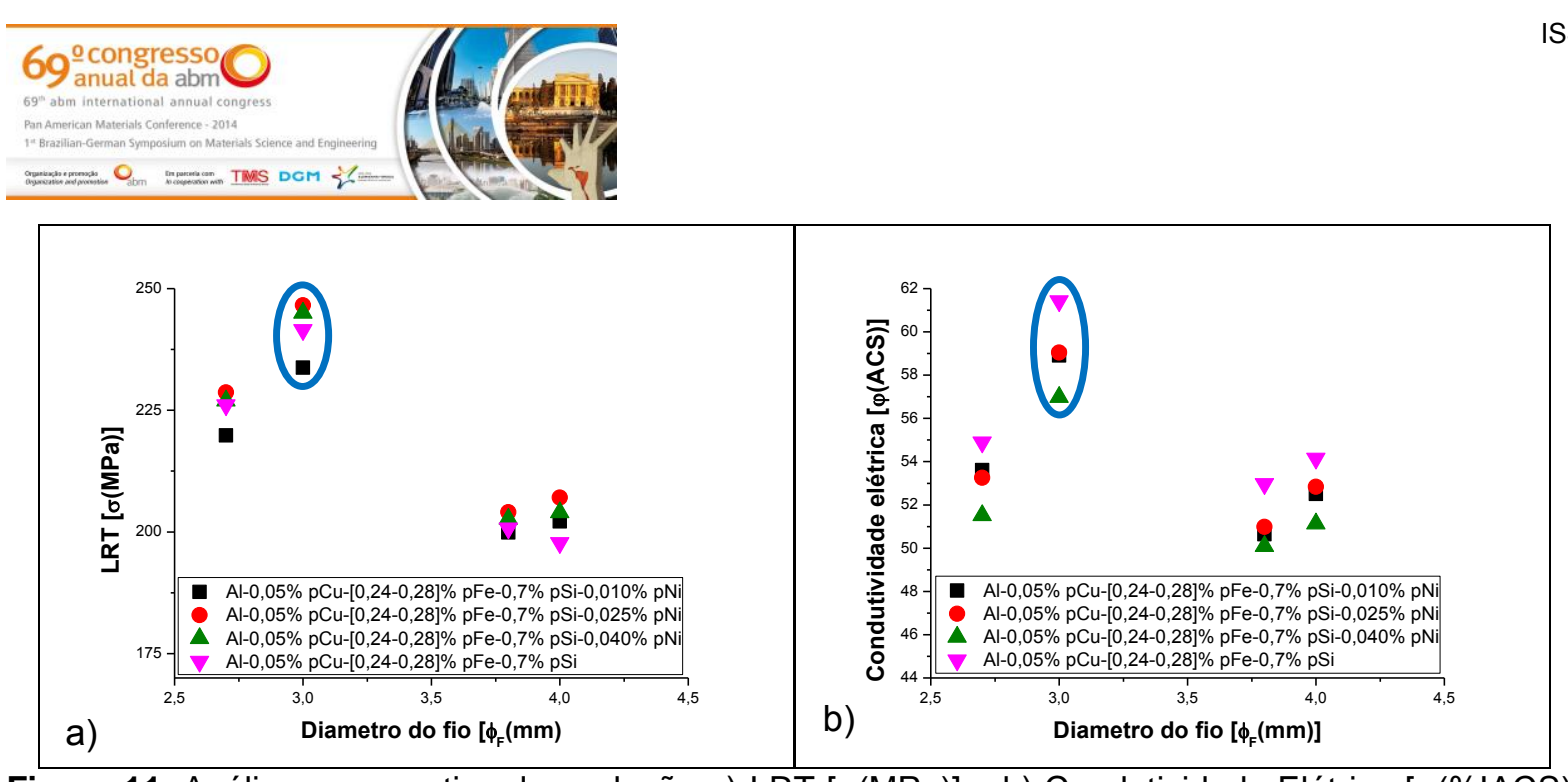

Figura 11. Análise comparativa da evolução a) LRT $[\sigma(\mathrm{MPa})]$ e b) Condutividade Elétrica $[\varphi(\% \mid A C S)]$ para as ligas para as ligas: Al-0,05\%Cu-[0,24-0,28]\%Fe-0,7\%Si; Al-0,05\%Cu-[0,24-0,28]\%Fe-0,7\%Si$0,010 \% \mathrm{Ni} ; \mathrm{Al}-0,05 \% \mathrm{Cu}-[0,24-0,28] \% \mathrm{Fe}-0,7 \% \mathrm{Si}-0,025 \%$ Ni e Al-0,05\%Cu-[0,24-0,28]\%Fe-0,7\%Si$0,040 \% \mathrm{Ni}$.

\section{CONCLUSÃo}

A presença de Ni melhora a capacidade de endurecimento de liga, mesmo em detrimento do teor $0,010 \% \mathrm{Ni}$ em alguns diâmetros.

A presença de Ni minimiza a capacidade de condução de energia elétrica.

O maior grau de deformação $\left(\varnothing_{\mathrm{F}}=3,0 \mathrm{~mm}\right)$ apresenta maiores resultados, tanto para a condutividade elétrica, como para o limite de resistência a tração, bem como apresenta menores resultados para 0 alongamento.

O teor de $0,025 \% \mathrm{Ni}$ foi o que apresentou melhor desempenho à deformabilidade, apresentando maiores limites de resistências mecânica para todos os diâmetros.

E por fim, os resultados obtidos e discutidos apontam para a liga com $0,025 \% \mathrm{Ni}$ e para o diâmetro de 3,0 $\mathrm{mm}$ como as melhores performances apresentadas por este trabalho.

\section{Agradecimentos}

Agradecemos ao CNPq pelo apoio concedido às pesquisas através da concessão de bolsas ao autor e coautores deste trabalho.

\section{REFERÊNCIAS}

1 ABAL. Soluções Sustentáveis. Associação Brasileira do Alumínio (ABAL). Disponível em: <http://www.abal.org.br/sustentabilidade/solucoes-sustentaveis/>. Acesso em: 15 mar. 2014.

2 O Setor Elétrico. Fios e cabos: condutores da evolução humana. Disponível em: <http://www.osetoreletrico.com.br/web/a-revista/edicoes/223-fios-e-cabos-condutoresda-evolucao-humana.html >. Acesso em: 25/11/13.

3 Bresciani EF, Zavaglia CAC, Button ST, Gomes E, Nery FAC. Conformação Plástica dos Metais. 4ª Ed. Unicamp, 1991, p.17.

4 Reed-Hill RE. Princípios de Metalurgia Física, Ed. Guanabara Dois, 2ª . edição, 1982.

5 Askerland D, Phulé P. Ciência e Engenharia de Materiais. Cap. 9, São Paulo, Cengage Learning, 2008.

6 Albuquerque VHC, Cortez PC, Alexandria AR, Aguiar WM, Silva EM. Sistema de segmentação de imagens para quantificação de microestruturas em metais utilizando redes neurais artificiais. Revista Matéria, 2007; 12(2): 394 - 407.

\footnotetext{
* Contribuição técnica ao 69ำ Congresso Anual da ABM - Internacional e ao 14ํㅡㄹ ENEMET - Encontro Nacional de Estudantes de Engenharia Metalúrgica, de Materiais e de Minas, 21 a 25 de julho de 2014, São Paulo, SP, Brasil.
} 


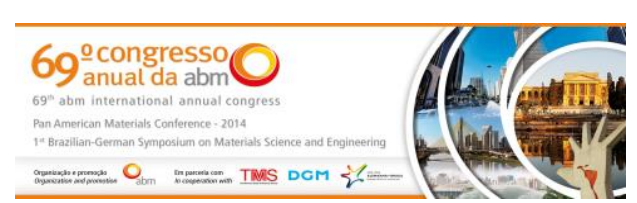

7 Narayanasamy R, Parthasarathi NL, Ravindran R, Sathiya Narayanan C. Analysis of fracture limit curves and void coalescence in high strength interstitial free steel sheets formed under different stress conditions. Springer Science Business Media, LLC 2008.

8 Narayanasamy R, Parthasarathi NL, Sathiya Narayanan C. Effect of microstructure on void nucleation and coalescence during forming of three different HSLA steel sheets under different stress conditions. Materialsand Design, 2008.

9 Ravindran R, Manonmanib K, Narayanasamy R. Analysis of void coalescence in AL 5052 alloy sheets annealed at different temperatures formed under different stress conditions. Materials Science and Engineering A, 2009.

10 Naga Krishna N, Akash A, Sivaprasad K, Narayanasamy R. Studies on void coalescence analysis of nanocrystallinecryorolled commercially pure aluminium formed under different stress conditions. Materials and Design, 2010.

* Contribuição técnica ao $69^{\circ}$ Congresso Anual da ABM - Internacional e ao 14ํㅡㄹ ENEMET - Encontro Nacional de Estudantes de Engenharia Metalúrgica, de Materiais e de Minas, 21 a 25 de julho de 2014, São Paulo, SP, Brasil. 